\title{
From metal ions to biospecies: template- assisted synthesis as a strategy to generate artificial receptor materials
}

\section{Peter A. Lieberzeit ${ }^{{ }^{\star}}$, Sadia Bajwa ${ }^{1}$, Ghulam Mustafa ${ }^{1}$, Thipvaree Wangchareansak ${ }^{2}$, Franz L. Dickert $^{1}$}

\author{
${ }^{1}$ University of Vienna, Department of Analytical Chemistry, Währinger Strasse 39, 1090 Vienna, Austria \\ ${ }^{2}$ Department of Chemistry, Faculty of Science, Kasetsart University, Chatuchak, Bangkok 10900, Thailand \\ *Corresponding author. E-mail: Peter.Lieberzeit@univie.ac.at
}

Received: 16 April 2011, Revised: 28 July 2011 and Accepted: 08 Aug 2011

\section{ABSTRACT}

Materials science increasingly focuses on generating "smart", i.e. highly functional, advanced matrices. Selective recognition can be implemented into man-made polymers by template-assisted synthesis. The method covers a surprising size range: it is possible to generate sensitivity and selectivity towards bivalent metal cations, such as $\mathrm{Cu}^{2+}$, in polyacrylate matrices. Despite the template being substantially smaller in size than the monomers, recognition can be achieved. Furthermore, acrylates can be templated with much larger species, such as influenza viruses. This leads to a nanostructured polymer surface that selectively rebinds the respective virion. Additional enhancement of sensitivity can be achieved by composite materials. Silver sulphide nanoparticles for instance show very appreciable affinity towards butanol vapors. When blending into butanol-imprinted polyurethane, the material incorporates three times more of the alcohol. Copyright @ 2011 VBRI press.

Keywords: Molecular imprinting; metal ion sensing; composite materials; virus imprinting; sensor.

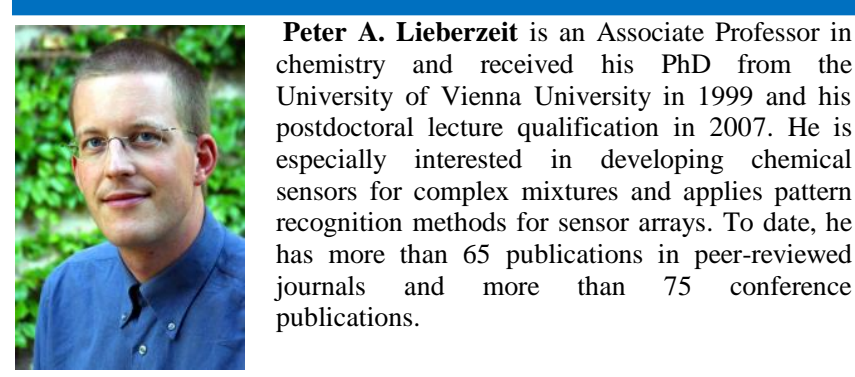

\section{Introduction}

Introducing self-organization approaches into materials synthesis opens up the way to implement surprising properties into matrices that would otherwise be regarded rather "conventional". Among others, such directed synthesis can be realized by (molecular) imprinting [1], where an organic or inorganic polymeric matrix is prepared in the presence of a template species directing the growth of the chain. In non-covalent imprinting [2] the template interacts with functional groups of monomers and thus generates a pre-formed network of interaction sites. After removing it, the remaining polymer matrix contains cavities in its bulk or on its surface and can be used for different applications including sensing, but for instance also drug release [3]. Within this paper, we present different approaches pushing the borders of imprinting into different directions concerning both the size of the respective template and the possibilities to implement composite material approaches.

The materials' interaction properties are the main focus of interest, because they inherently also determine their application. Aside of well-established instrumental techniques ranging from spectrometry to scanning microscopy, we also characterize them by applying them as artificial receptors for chemical sensing, mainly by combining their recognition abilities with the masssensitivity of e.g. quartz crystal microbalances (QCM) or with interdigital electrode structures (IDC) yielding contactless conductivity/capacity signals.

\section{Results and discussion}

Imprinting with metal ions

Molecular imprinting has attracted substantial interest during the last two decades, but still many fundamental questions remain open. Whereas the current upper size limit of imprinting is determined by pollen with a diameter of $25 \mu \mathrm{m}$ [4], the minimum size of a template is most probably determined by the diameters of metal ions [5]. In the present study we therefore targeted at designing materials 
selectively incorporating bivalent metal ions, viz. copper ions, for their selective detection in aqueous media. For this purpose, we synthesized acrylate-based cross linked copolymers directly on different transducers, both as-is and in the presence of the template. Fig. 1 shows the responses of both a QCM and a interdigital capacitor, coated with both the non-imprinted (NIP) and imprinted (MIP) material, respectively, and exposed to solutions containing $1 \mathrm{mM} \mathrm{Cu}^{2+}$. As can be seen, in both cases the NIP material gives rise to but very minor effects. In contrast to this, the MIP in both cases gives rise to substantial sensor responses: in the case of the QCM, the signal is $2.5 \mathrm{kHz}$, in the case of IDC the conductance increases by almost an order of magnitude. Therefore, the presence of the ion during templating substantially influences the morphology of the respective polymer matrix, which is rather surprising given the fact that metal ions are much smaller in size than each of the monomer components used. Furthermore, the non-imprinted polymer has completely masked the influence of the copper on the IDC and shows similar behavior on the QCM. Hence, choosing the correct monomers can lead to selective recognition even in the case that they are much larger than the target species. Furthermore, it strongly underpins the role of the template, as can be seen from the substantial difference in response between the two materials. However, the QCM results with MIP suggest that the quartz does not only react to the mass change on the surface, but also to changes in electrical conductivity of the surrounding medium.
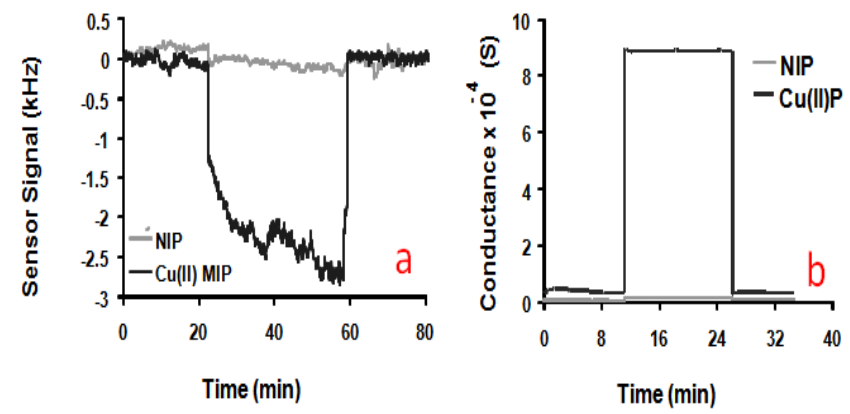

Fig. 1. QCM and IDC sensor responses for $\mathrm{Cu}(\mathrm{II})$-imprints and the nonimprinted counterpart, respectively.

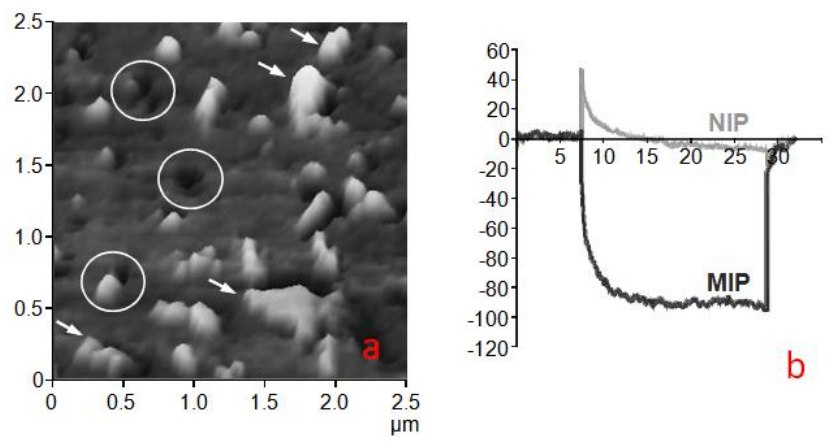

Fig. 2. (a) AFM image of Influenza Virus MIP with partly filled cavities; (b) Influenza virus MIP and NIP sensor responses with QCM

\section{Influenza virus MIP}

Templates are of course not limited to molecular compounds but can be as large as entire biospecies. Viruses are especially interesting in this case, because due to their dimensions of some ten nanometers in diameter there is currently no possibility for their rapid on-line detection. In this case recognition materials are available via surface imprinting techniques, where the species of interest is pressed into the surface of a pre-polymerized material. In such a way it is possible to generate surface recognition sites within acrylate copolymers to interact with (deactivated) influenza viruses. Fig. 2a) shows the result of such an approach: here a polymer surface can be seen after templating with $\mathrm{H} 1 \mathrm{~N} 3$ virus strain. The templates have been partially removed; therefore the surface shows both the cavities caused by the imprinting procedure (marked by circles) and the viruses that are still bound in these sites (marked by arrows). The elevations in the AFM image are indeed caused by virions, as can be judged from the diameter of roughly $70 \mathrm{~nm}$, which corresponds to the actual size of influenza viruses. After washing, the resulting materials exhibit sensitive properties, as can be seen in Fig. 2b: whereas the non-imprinted material again only shows minor effects, the virus MIP readily incorporates influenza It has to be noted that the chemical composition of the polymer used has some similarity to the one utilized for cation imprinting, which clearly shows the influence of the templating procedure. It generates artificial receptors in the bulk or on the surface of a polymeric matrix, respectively, and determines the interaction properties of the final material over a size range of almost three orders of magnitude in the concrete case.

\section{Composite materials}

Generally, the sensitivity of a sensor can be increased by generating the maximum possible number of interaction sites within the sensor material and/or by increasing the accessibility of the said interaction centers [6]. Furthermore, sensitivity limitations can also be overcome by introducing some sensor materials with a sufficient affinity interaction with analyte [7]. When bringing the two approaches together, the combination of organic and inorganic nanosized materials can for instance result in a nano-composite material with novel collective mechanical, thermal, optical, magnetic and electronic properties $[8,9]$. As an example, we have introduced chemically selective $\mathrm{Ag}_{2} \mathrm{~S}$ nanoparticles (NP) into a polyurethane layer to achieve high sensitivity by blending the affinity properties of the silver sulphide with the MIP within a single sensor layer. The outcome of this approach can be seen in Fig. 3 summarizing the QCM sensor responses in gas phase of both the pure MIP material and the composite when exposed to air streams with defined butanol concentration. Obviously, the composite takes up around three times more butanol than the pure MIP of the same layer height. This can be traced back to two different effects: on the one hand, it is caused by the affinity interaction of the analyte (1butanol) with $\mathrm{Ag}_{2} \mathrm{~S}$ nanoparticles present in composite. On the other hand the reason is the increased availability of interaction sites: a diffusion pathway on the average is shorter in the case of the affinity material due to the 
enhanced surface area of nanoparticles as compared to simple polymer layer. Therefore, the composite material seems to be promising candidate as a recognition material for alcohol detection by interacting reversibly.

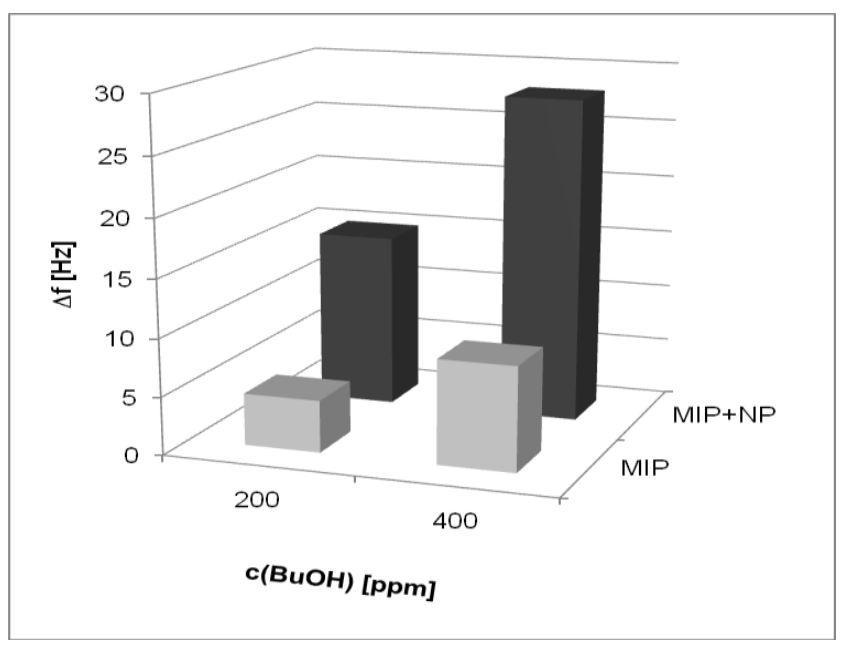

Fig. 3. QCM sensor responses of $\mathrm{BuOH} \mathrm{MIP}$ and $\mathrm{MIP} / \mathrm{Ag}_{2} \mathrm{~S}$ nanoparticlecomposite material, respectively.

\section{Conclusion}

Functionality suitable for chemical sensing can be generated on a variety of size scales ranging from Angstrom to the high nanometer range and beyond. The interactions involved include affinities based on the hardsoft principle, polar and hydrogen bonds and $\pi-\pi$-interactions among others. The task of materials chemistry is to achieve the necessary fine tuning.

\section{Reference}

1. Karim, K; Breton, F; Rouillon, R.; Piletska, E. V.; Guerreiro, A.; Chianella, I.; Piletsky, S. A. Adv. Drug Deliv. Rev. 2005, 571795. DOI: $10.1016 /$ j.addr.2005.07.013

2. Ansell, R. J.; Kriz, D.; Mosbach, K. Curr. Opin. Biotechnol. 1996, 7, 89.

DOI: $\underline{10.1016 / \mathrm{S} 0958-1669(96) 80101-7}$

3. Li, S.; Tiwari, A.; Ge, Y.; Fei, D. Adv. Mater. Lett. 2010, 1, 4 DOI: $10.5185 /$ amlett.2010.4110

4. Jenik, M.; Seifner, A.; Lieberzeit, P.; Dickert, F. L. Anal. Bioanal. Chem. 2009, 394, 523. DOI: $10.1007 / \mathrm{s} 00216-009-2718-8$

5. Rao, T. P.; Kala, R.; Daniel, S. Anal. Chim. Acta 2006, 578, 105. DOI: $10.1016 /$ j.aca.2006.06.065

6. Di Natale, C.; Paolesse, R.; Macagnano, A.; Nardis, S.; Martinelli, E.; Dalcanale, E.; Costa, M.; D’Amico, A. J. Mater. Chem. 2004, 14 , 1281.

DOI: $10.1039 / \mathrm{b} 313250 \mathrm{a}$

7. Lieberzeit, P. A.; Afzal, A.; Rehman, A; Dickert, F. L. Sens. Actuators B 2004, 127, 132.

DOI: $10.1016 / \mathrm{j} . \mathrm{snb} .2007 .07 .020$

8. Fang, Y.; Bai, Ch.; Zhang, Y. Chem. Commun. 2004, 804. DOI: $10.1039 / \mathrm{B} 314533 \mathrm{~F}$

9. Chan, Y. N.; Craig, G. S. W.; Schrock, R. R.; Cohen, R. E. Chem. Mater. 1992, 4, 885 .

DOI: $10.1021 / \mathrm{cm} 00022 \mathrm{a} 026$

\section{Acknowledgements}

This work was partly financially supported by the Pakistan Overseas Scholarship Program for $\mathrm{PhD}$ and the Royal Golden Jubilee Grant Program, Thailand.

\section{AdVAnCed Materials Letters}

\section{Publish your article in this journal}

ADVANCED MATERIALS Letters is an international journal published quarterly. The journal is intended to provide top-quality peer-reviewed research papers in the fascinating field of materials science particularly in the area of structure, synthesis and processing, characterization, advanced-state properties, and applications of materials. All articles are indexed on various databases including $\mathrm{DOAJ}$ and are available for download for free. The manuscript management system is completely electronic and has fast and fair peer-review process. The journal includes review articles, research articles, notes, letter to editor and short communications.

Submit your manuscript: http://amlett.com/submitanarticle.php

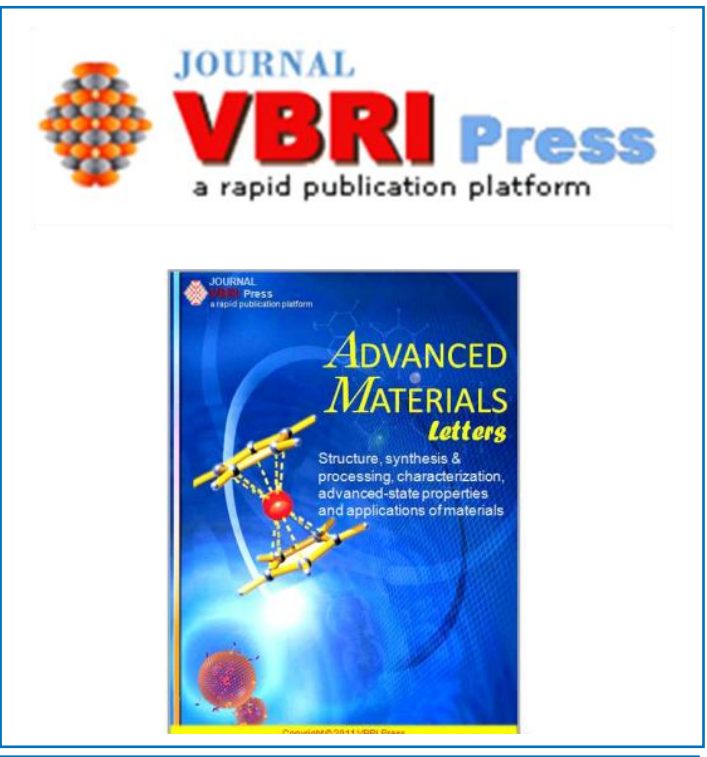

